\title{
Advantages of E-Health System Management
}

\section{Gaurav Bordoloi and Sahadev Roy}

\begin{abstract}
The main objectives of E-health system are, those people, suffering from specific diseases which may create critical health situation, need continuous monitoring of health. This health care system provides real time monitoring system. An autonomous embedded system is a solution for continuous monitoring of person's health. A patient can be monitored from anywhere and data can be transmitted to the care centre where experts can keep track of it. Specialized computing devices are used for processing, transportation, archiving and visualization of acquired data. Here we introduce mobile ambulance, E-card, smart ICU and online doctor association. In this paper, we discuss the various advantages of E-health system.
\end{abstract}

\section{Keywords}

E-Health;

E-Cards;

Embedded System;

Mobile Ambulance;

Online Doctors Association;

Smart ICU.

\section{INTRODUCTION}

The development of any country depends on the wellbeing of its citizens so good health is a necessity of every one. A country with its healthcare system defines its Human development index [1]. Number of people requiring frequent medical check-ups and examinations is increasing day by day. Developing countries and economic powerhouse countries like India still lag behind in its Human Development index ranked at 131(2017) [2] due to less advancement in healthcare systems. Now, everything is converting into smartness due to advancement in automation and artificial intelligence i.e. irrigation system, home automation, security system, health care system etc. As technologies are increasing in modern life style, modern diseases are also on an increase in human society. Diseases like Ebola and Zika were unheard about in the past; drug resistance and strain evolution are the main reasons for this [3]. Automation in medical development has led to various systems being developed to diagnose and treat diseases. There has been furious growth in interest on embedded system for biomedical application in last few years which is increasing the demand of computing and communication, but at the same time, encouraging the necessity to keep those applications portable and autonomous. Portability and logistics are the main things to be considered for such developments as such system relies on efficient management and transportation [4]. Clinical analysis, proteomics analysis, and real-time patient monitoring and biomedical assistants are the example where portability is required. For this portable system to reach the nook and corners of society logistics is an issue. Communication with general purpose computing systems leads to the integration of wireless devices on the embedded systems. Therefore, embedded systems have to be designed with low power communication subsystems for biomedical applications and have to be easily integrated with more general distributed computing platforms [5]. At computation and communication levels, reliability and security issues have to be considered on those platforms [6]. Systems should also be designed to take into account the overall affordability of the setup.

\section{IMPLEMENTATION OF E HEALTH MANAGEMENT}

With modern economical and advancing technology, an implementation of a smart mobile Internet of Things (IoT) system is proposed which is aimed at monitoring patients through the internet. This system will provide updated health statistics to doctors and nurses connected over the internet and thus monitor and provide immediate response. This system, based on Arduino will monitor statistics like body temperature, pulse and heart-rate, surrounding temperature thus providing an almost all round monitoring of the patient.

\section{A. Smart e-Ambulance}

In every critical situation, the patient needs to call an ambulance but in the e-health care system there is an auto location detector is available and that is able to sense the location of the patient [7] and locate the hospital in that range of area and if the distance is more than the threshold then first it updates the patient that he/she needs to go to the hospital whether there is a need of ambulance or not and if the response is positive it calls the nearby ambulance. A link is maintained between these 
groups of ambulances which serve to the purpose of mobile ambulance so that they contact from time to time and maintain constant contact with each other [8]. Ambulance may also be provided a wireless communication system in which the alarm signal is be sent to an ambulance service in case of emergency situation in which the patient require immediate treatment and assistance [9]. The ambulance also have a communication system tied up with the hospitals as to send the complete report of patient before the patient reaches the hospital so that the mentioned time for starting the patient treatment can be reduced as much as possible by pre diagnosing the cause and condition of the patient thus saving precious time for faster treatment and recovery.

\section{B. E-Helth Card}

In e-health care module whenever a patient is being admitted for the treatment, an electronic card with unique ID is prepared and it is given with all the information about patient previous health statistics and information related to disease diagnosis [10]. Ecards are electronically configurable system on a card that provides all details about patient beforehand to hospitals and can be carried with the patient [11]. Further test reports and the treatment related information can be stored in that e-card and on the central web server. It is helpful in that sense as the person is referred to any other hospital and at that time the card will provide all the history of the patient treatment as well as all the diagnosis information. If the card is missed in the case of hurry all the information can be retrieved by the central web server by that unique ID [12].

\section{Online Doctors Association}

As the complete system is connected to the central server the reports of the patient are being sent to the doctors or experts who are available online at that time for medical services [13]. As the report are received by the doctors they prescribe the patient all the first aids before going to the hospital, this will reduce the problems of the patient as well as fast track the recovery process. If the available doctors cannot handle the problem the system will send that report to the experts and then the experts respond according to the requirement [14]. Thus an organic linkage is maintained by the system and the association of the doctors. This online health care is also helpful for the students of medical institutions as they can explore their knowledge by reading all the condition which arrives in real time.

The overall Framework Architecture of E Health Management is shown in Fig 1.

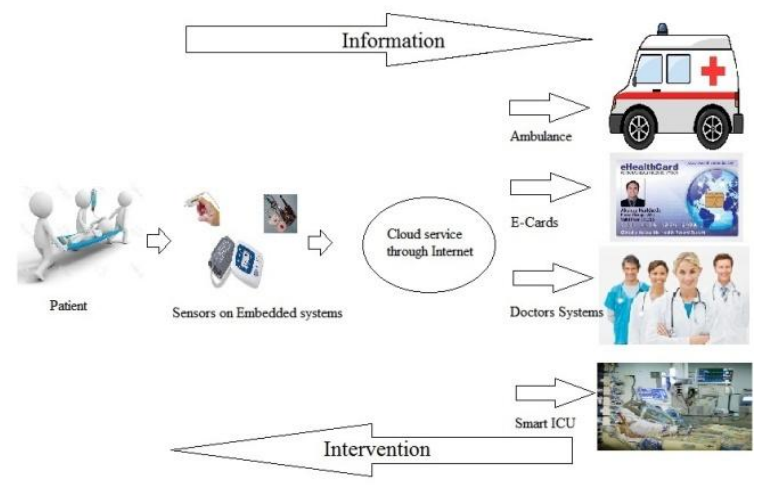

Fig 1. Framework Architecture of $E$ Health Management

\section{Advantages of E-Health Care} SYSTEM

As in conventional system the paper prescription was given so there is a possibility of losing that prescription and the medicines cannot be taken on time, this type of problems are reduced by this ehealth care system. As the e-health care system introduces the doctors can directly communicate with pharmacist so that the error in understanding the medicines will reduce. By using $\mathrm{E}$ health system patient can directly contact an ambulance in case of an emergency or critical situation and direct get help also can directly communicate with doctor and that will also reduce time from the conventional methods such as apply for appointment and all such other methods. Thus E-Health cards come as a saviour in such situation. At the time of emergency every second is very important and it makes a difference between life and death so this method saves the time.

In traditional health monitoring system all the details related to the patient are written on paper and stored in the database of the hospital where the patient is treated and as the time goes on there is a possibility of missing that data and also in some cases if patient has suffer from the same problem again or any new situation caused and if he/she consulted to a new doctor or the new hospital doctor faces the difficulty because they are unware about patient's previous conditions. So as the digital 
database is created this type of problem will not appear [15]. The doctors can easily be made aware about the patient's previous health record and it will help during diagnosis by providing right diagnosis and faster healing. In situation of any direful event, people usually get confused and frightened so there is a possibility of forgetting about the previous medical details.

Accurate, up to date and full information gives the very high quality of health care with reduced number of errors during diagnoses [16]. In this design, reminders are send by electronic system so it is helpful in providing the real time updates of the system, by this patient can take care of himself [17]. This e-health care system is better than the previous used traditional health care system as it is quickly access the information of patient from any location with specified queries also.

\section{CONCLUSION}

Cryptosystems and unique ID can also be used to assure the security when any data needs to be transmitted. The challenges of future could be to innovate and invent the constraints and the compact devices for faster, smarter, reliable and very user friendly the use of E-health systems. Thus e-cards can come as a life saviour in situation in which patient may not remember details of previous ailments and diagnostics and advanced drugs can be prescribed to him in case of situation in which previously prescribed drugs do not inhibit the control of the disease of which he is suffering and also eliminate the chances and risk of wrong diagnosis and drugs. Interchange between information among clinicians, experts, diagnostic and pharmacies center is trackable and faster by which the reduction in probability of losing the information and following calls. These features of e-health care leads to the greater productivity and efficiency in the world of medical science.

\section{REFERENCES}

[1] Anderson, G. F., Reinhardt, U. E., Hussey, P. S., \& Petrosyan, V. (2003). It's the prices, stupid: why the United States is so different from other countries. Health Affairs, 22(3), 89-105.

[2] Elizabeth Roche. (2017, Mar.) India ranks 131 on Human Development Index, Norway No.1. [Online].

http://www.livemint.com/Politics/NcyY1Zr768
TEl02yaRSh4M/India-ranks-131-on-globalHuman-Development-Index-Norway-No.html

[3] Davies, J., \& Davies, D. (2010). Origins and evolution of antibiotic resistance. Microbiology and molecular biology reviews, 74(3), 417-433.

[4] Atzori, L., Iera, A., \& Morabito, G. (2010). The internet of things: A survey. Computer networks, 54(15), 2787-2805.

[5] Chen, M., Wan, J., González-Valenzuela, S., Liao, X., \& Leung, V. C. (2014). A Survey of Recent Developments in Home M2M Networks. IEEE Communications Surveys and Tutorials, 16(1), 98-114.

[6] Pottie, G. J., \& Kaiser, W. J. (2000). Wireless integrated network sensors. Communications of the ACM, 43(5), 51-58.

[7] Duffin, E. G., Thompson, D. L., Goedeke, S. D., \& Haubrich, G. J. (1998). U.S. Patent No. 5,752,976. Washington, DC: U.S. Patent and Trademark Office.

[8] Tjora, A. (2004, November). Maintaining redundancy in the coordination of medical emergencies. In Proceedings of the 2004 ACM conference on Computer supported cooperative work (pp. 132-141). ACM.

[9] Donaldson, M. S., Corrigan, J. M., \& Kohn, L. T. (Eds.). (2000). To err is human: building a safer health system (Vol. 6). National Academies Press.

[10] Borgia, E. (2014). The Internet of Things vision: Key features, applications and open issues. Computer Communications, 54, 1-31.

[11] Shortliffe, E. H., \& Cimino, J. J. (Eds.). (2013). Biomedical informatics: computer applications in health care and biomedicine. Springer Science \& Business Media.

[12] Clarke, I., Sandberg, O., Wiley, B., \& Hong, T. W. (2001). Freenet: A distributed anonymous information storage and retrieval system. In Designing privacy enhancing technologies (pp. 46-66). Springer Berlin Heidelberg.

[13] Andrews, Lori B., et al. An alternative strategy for studying adverse events in medical care. The Lancet 349.9048 (1997): 309-313.

[14] Ash, J. S., Berg, M., \& Coiera, E. (2004). Some unintended consequences of information technology in health care: the nature of patient care information system-related errors. Journal of the American Medical Informatics Association, 11(2), 104-112. 
[15] Tongdee, Trongtum, and Vamsidhar Narra. Medical digital asset management system and method. U.S. Patent Application No. 11/081,254.

[16] Shneiderman, B. (2010). Designing the user interface: strategies for effective humancomputer interaction. Pearson Education India.

[17] Barrick, I. J. (2009). Transforming Health Care Management: Integrating Technology Strategies: Integrating Technology Strategies. Jones \& Bartlett Publishers

\author{
Authors \\ Gaurav Bordoloi \\ Dept. of ECE, \\ NIT Arunachal Pradesh \\ Email: gauvhs@gmail.com \\ Sahadev Roy \\ Dept. of ECE, \\ NIT Arunachal Pradesh \\ Email: sdr.ece@nitap.in
}

